\title{
Endoscopic transmural drainage combined with percutaneous drainage in treatment of walled-off pancreatic necrosis-case report
}

\author{
Mateusz Jagielski*1, Marian Smoczynski ${ }^{1}$, Michał Studniarek ${ }^{2}$, Krystian Adrych $^{1}$ \\ ${ }^{1}$ Department of Gastroenterology and Hepatology, Medical University of Gdansk, Gdansk, Poland \\ ${ }^{2}$ Department of Radiology, Medical University of Gdansk, Gdansk, Poland
}

\begin{abstract}
Received: January 6, 2016
Accepted: February 13, 2016

Online Published: February 23, 2016

DOI: $10.5430 /$ crim.v3n2p1

URL: http://dx.doi.org/10.5430/crim.v3n2p1

Abstract

Pancreatic necrosis and the necrosis of surrounding tissues are found to be local complications occurring in $15 \%$ of patients suffering from acute pancreatitis. Walled-off pancreatic necrosis (WOPN) is in fact pancreatic fluid collection surrounded by a well-defined wall and inclusive of liquefied necrosis and elements (debris) of necrotic tissues. Endotherapy is an efficient method of treatment for patients with symptomatic WOPN. Often single transmural access to the necrotic collection is sufficient. However, in some patients with infected WOPN, the application of an extra way of access to pancreatic necrosis is a must. In these particular patients the optimal strategy is joining a few minimally invasive methods, which allows multiplexing of access to the collection. Herein we described the case of treatment with the use of minimally invasive techniques in a patient suffering from an infected WOPN, in which the single transmural access to the necrotic collection appeared to be insufficient and the application of an extra way of access to pancreatic necrosis was necessary. Only joining together both minimally invasive techniques-transmural drainage and percutaneous drainage had become efficient enough and led to complete healing of the patient.
\end{abstract}

Key Words: Endoscopic drainage, Transmural drainage, Percutaneous drainage, Walled-off pancreatic necrosis, Acute pancreatitis

\section{INTRODUCTION}

Acute Pancreatitis (AP) is considered to be an inflammatory process involving the pancreas as well as surrounding tissues and distant organs as the disease develops. The course of AP differs a lot from local inflammation into systemic inflammatory response syndrome (SIRS) running with multiple organ dysfunction. ${ }^{[1,2]}$ According to the revision of classification from Atlanta 2012, acute pancreatitis has been used to be divided into two morphological types-interstitial edematous AP and necrotizing AP. ${ }^{[1,2]}$ In the first type en- largement of the pancreas caused by inflammatory edema is displayed on imaging examination. ${ }^{[1-3]}$ The second type of AP is specified by necrosis involving parenchyma of the pancreas, tissues surrounding the pancreas or both structures at the same time ${ }^{[1,3]}$.

The course of acute pancreatitis may go as far to a rise of local consequences in the form of pancreatic and peripancreatic fluid collections. Four types of such fluid collections are distinguished depending on the period of the disease and the morphological type of AP: acute peripancreatic fluid collec-

\footnotetext{
*Correspondence: Mateusz Jagielski; Email: matjagiel@ gmail.com; Address: Department of Gastroenterology and Hepatology, Medical University of Gdansk, Gdansk, Poland.
} 
tion (APFC), pancreatic pseudocyst, acute necrotic collection $(\mathrm{ACN})$ and walled-off pancreatic necrosis (WOPN). ${ }^{[1,2]}$

The rise of acute necrotic collection is possible in connection with the course of acute necrotizing pancreatitis. The ACN present in the first four weeks of the disease includes variable amounts of fluid and necrotic tissue. ${ }^{[1]}$ The walled-off pancreatic necrosis occurs in a later period of acute necrotizing pancreatitis (after four weeks of the disease) and in fact is a preserved acute necrotic collection, surrounded by a well-defined wall and inclusive of liquefied necrosis and elements (debris) of necrotic tissues. ${ }^{[1,2]}$ The amount of debris in the lumen of the collection depends on the degree of liquidation, which further depends on the time elapsed from the beginning of the disease. ${ }^{[1,2]}$

The surgical procedures had been used to be the only method of treatment of pancreatic necrosis. Applied procedures of open necrosectomies are found to be burdened with high morbidity and mortality. ${ }^{[4-6]}$ Due to the development of minimally invasive techniques of treatment of pancreatic necrosis the access to the necrotic collection is possible through transperitoneal, retroperitoneal, transmural and transpapillary way. ${ }^{[7,8]}$ The optimal strategy in many patients is joining a few minimally invasive methods, which allows multiplexing of access to the collection. ${ }^{[7,9]}$ The randomized trial certified that the application of minimally invasive techniques in WOPN's treatment ("the step-up approach") significantly reduces the rate of complications and mortality compared to open a necrosectomy procedure. ${ }^{[10]}$

Herein we describe a case of treatment with use of minimally invasive techniques in a patient suffering from WOPN, in which the single transmural access to the necrotic collection appeared to be insufficient and the application of an extra way of access to pancreatic necrosis was necessary.

\section{Case presentation}

A 37-year-old patient was admitted to the Department of Gastroenterology and Hepatology of the Medical University of Gdansk due to alcoholic acute pancreatitis. On admission the patient was dehydrated with features of acute kidney failure and toxic hepatic injury. An intensive liquid therapy had been applied together with analgesic treatment and a starvation diet, after 5 days of which the enteral nutrition was implemented and continued for the next 14 days. In contrastenhanced computed tomography (CECT) of the abdomen an evolution of inflammatory changes in the pancreatic area with creation of an extensive area of necrosis running down the abdominal cavity up to the minor pelvis were observed.

On the 21 st day of the disease a fever reaching up to $39^{\circ} \mathrm{C}$ appeared together with increased levels of inflammatory markers profiled in laboratory blood tests (leukocytosis $23 \mathrm{G} / \mathrm{L}$, CRP level $223 \mathrm{mg} / \mathrm{L}$ ). The blood culture proved negative. The presence of fluid collection with gas bubbles and heterogeneous content indicating the presence of necrotic elements of the size $330 \mathrm{~mm} \times 125 \mathrm{~mm} \times 90 \mathrm{~mm}$ were revealed in next CECT of the abdomen. The described pancreatic fluid collection was starting at the infradiaphragmatic area, was running down the abdominal cavity and was ending at the minor pelvis.

An infected WOPN was recognized (see Figure 1). An antibiotic therapy was applied intravenously (Metronidazole, Piperacillin and Tazobactam). The patient was qualified to the endoscopic transmural drainage of WOPN.

In the fifth week of the disease a gastropancreatic fistula on top of the visible impression on the rear stomach wall was performed under endoscopic ultrasonography (EUS) control (see Figure 2). An outflow of dark-brown content with fragments of necrotic tissues through the stoma was observed. The gastropancreatic fistula was dilated with the use of a high pressure balloon up to $10 \mathrm{~mm}$. Two double pigtail $7 \mathrm{Fr}$ and 10 Fr stents were led through the stomy into the lumen of necrotic cavity together with nasocystic 8 Fr drain (see Figures 3, 4) in order to irrigate the collection (200 $\mathrm{ml}$ saline solution every four hours).

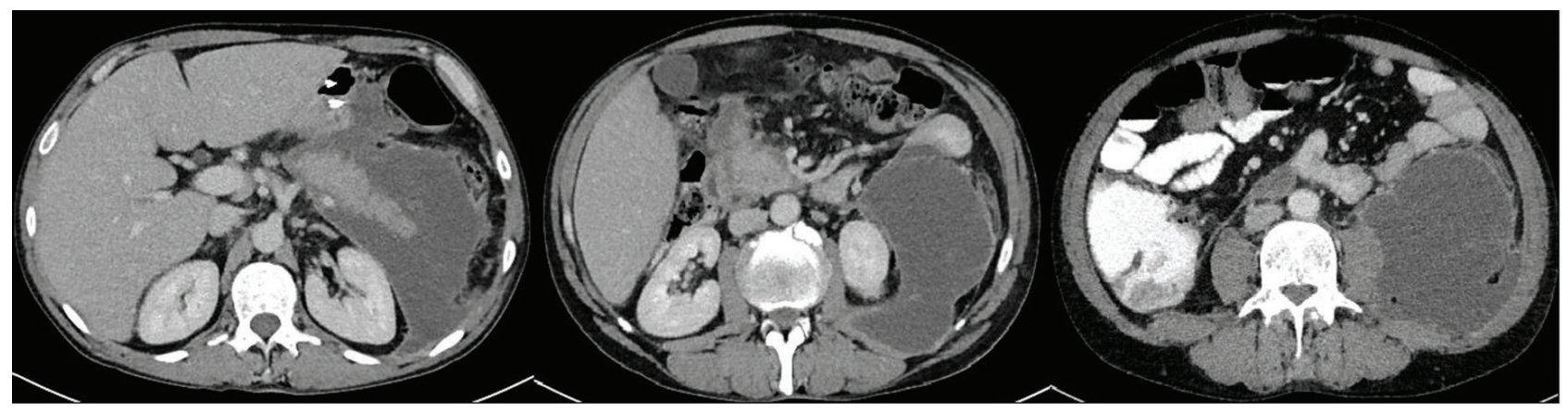

Figure 1. The CECT performed before the beginning of interventional treatment 


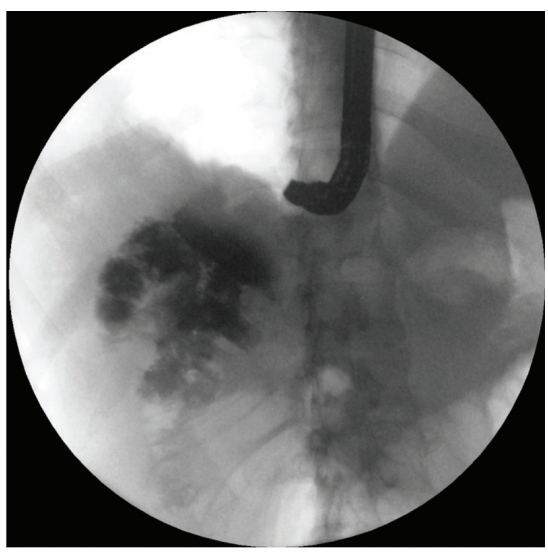

Figure 2. The endoscopic transmural drainage. Contrast medium being injected via catheter during the first endoscopic procedure fills the irregular cavity of necrosis's collection with numerous fragments of necrotic tissues in the lumen

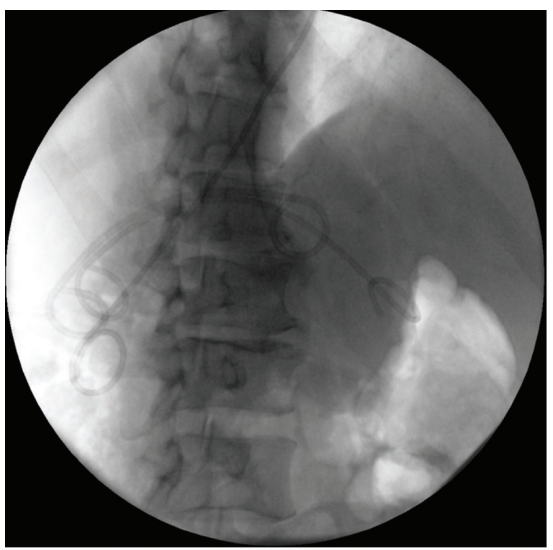

Figure 3. The endoscopic transmural drainage. The stents and the nasal drain guided through a stomy to the lumen of collection are evident

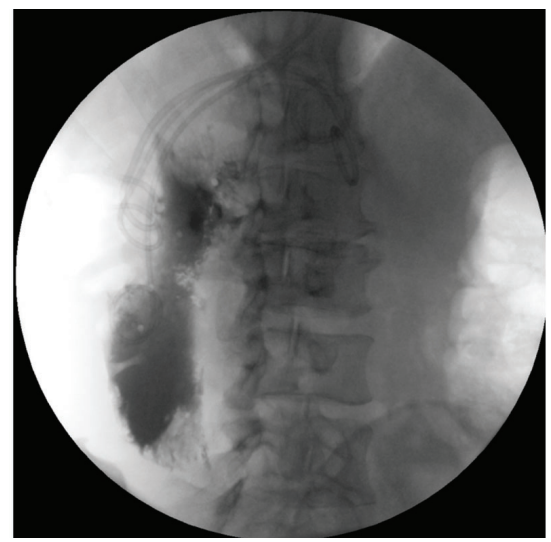

Figure 4. The endoscopic transmural drainage. The contrast medium injected via nasal drain fills the necrosis collection's cavity

The performed culture of the collection's content showed the presence of Enterococcus faecalis, Enterococcus faecium and Escherichia coli. Antibiotic therapy was continued for next 38 days in accordance with the culture of the collection's content.

Conventional ultrasonography was performed every 7 days in order to measure the efficiency of the treatment. Gradual regression of the necrotic collection was observed (see Figure 5). An endoscopic retrograde pancreatography (ERP) was done after 7 days of active transmural drainage. Complete disruption of the main pancreatic duct in the area of the tail of the pancreas was recognized (see Figure 6). A pancreatic 7 Fr endoprosthesis was guided transpapillary and it's distal ending was left in the pancreatic tail to bridge the damaged area of the duct.

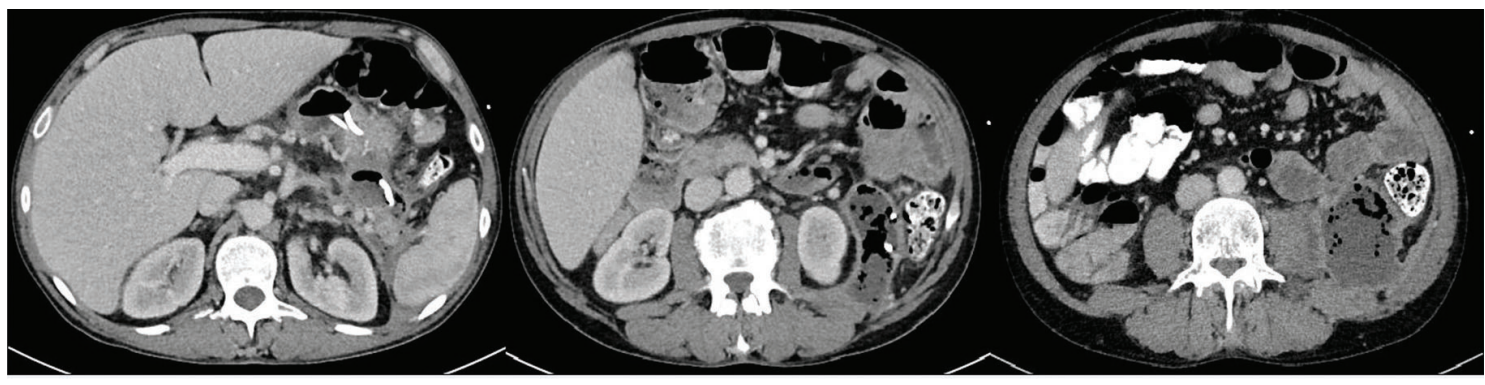

Figure 5. The CECT performed during minimally invasive treatment. A partial regression of WOPN is within sight. The nasal drain and transmural stents are noticeable in the lumen of necrotic collection

During the next endoscopic procedures gastropancreatic fistula was widened up to $20 \mathrm{~mm}$, two extra nasocystic $7 \mathrm{Fr}$ drains were put into the lumen of the collection and transmural stents were exchanged four times. Moreover, percutaneous drainage of WOPN was being performed for 25 days (see Figure 7).

Considering the absence of clinical symptoms and observed gradual regression of necrotic collection after 72 days of active drainage it was decided to remove the nasocystic drain 
leaving transmural and transpapillary stents.
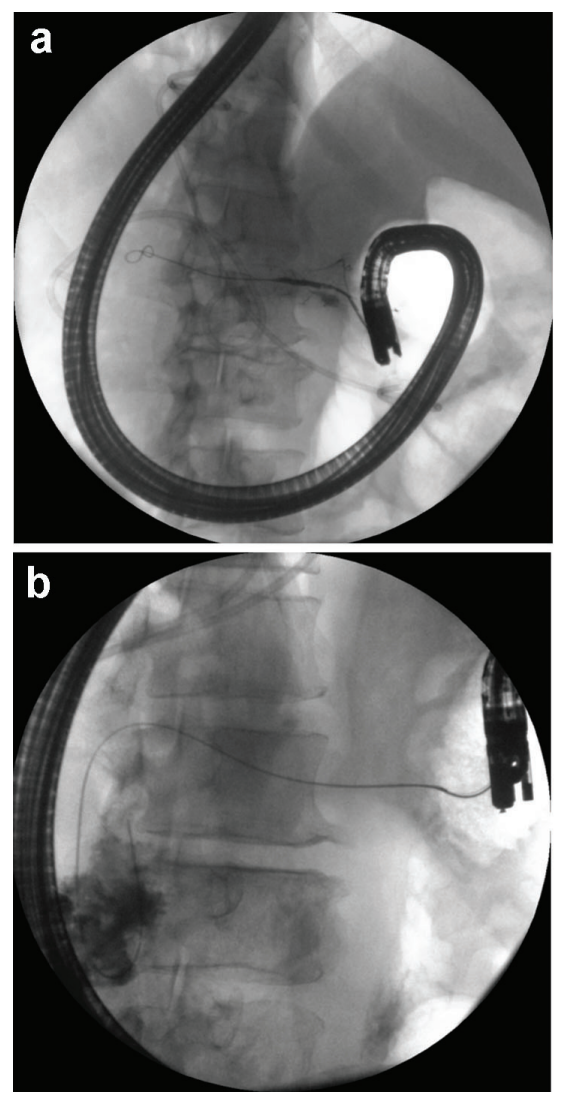

Figure 6. a: The endoscopic retrograde pancreatography reveals complete pancreatic duct's disruption in the area of pancreatic tail; b: The endoscopic retrograde pancreatography. A guide-wire introduced into the main pancreatic duct loops in the cavity of necrosis collection

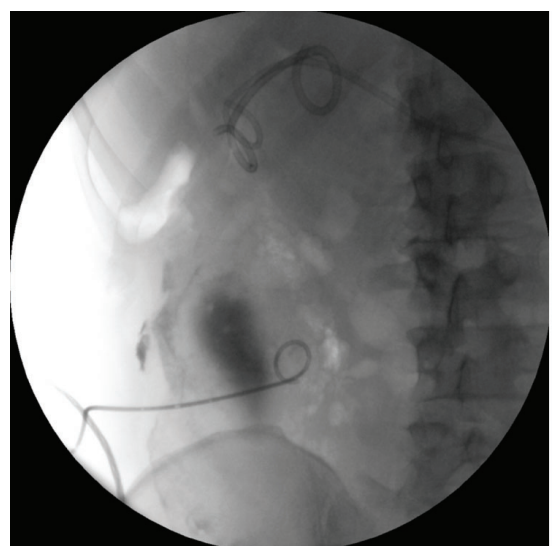

Figure 7. Draining system of WOPN. The stents, the nasal drain led transmurally and the percutaneous drain are noticeable. The contrast applied through the percutaneous drain filled the collection of pancreatic necrosis

During the next hospitalization and after three months from the end of active drainage, the complete regression of WOPN was stated in control abdominal CECT (see Figure 8). Then it was decided to remove the transmural stents. During the endoscopic procedure the pancreatic endoprosthesis was exchanged due to the presence of disruption of the pancreatic duct.

After a year of observation the transpapillary stent was also removed, because there was no observation of a leak of contrast outside the pancreatic duct during endoscopic retrograde pancreatography. At present the patient is in good general condition and has returned to full physical fitness and everyday activities.

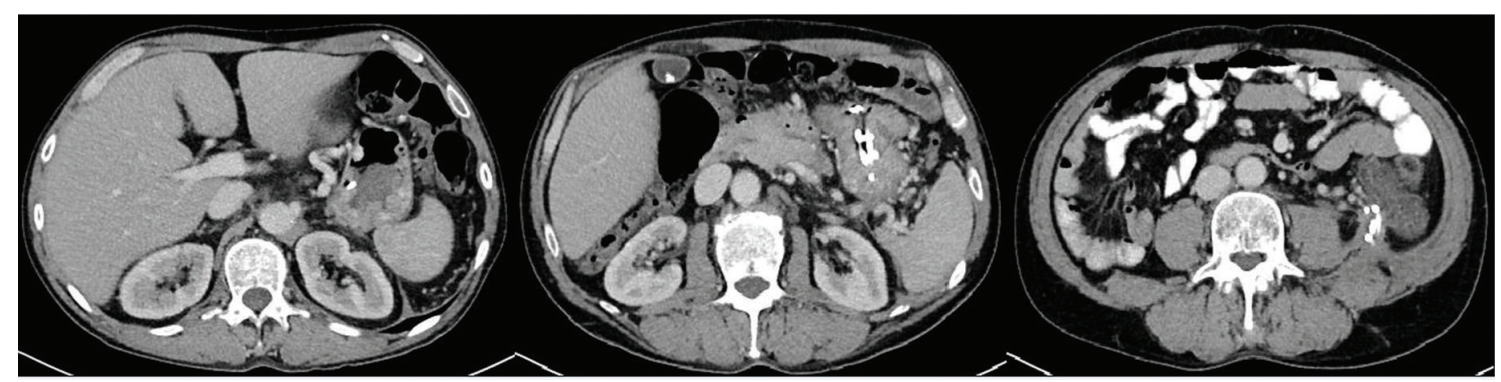

Figure 8. The CECT executed in the end of treatment. The complete regression of walled-off pancreatic necrosis is observed (collection less than $3 \mathrm{~cm}$ ). The transmural endoprosthesis are visible in the lumen of collection

\section{Discussion}

In the last twenty years a breakthrough in treatment of acute necrotizing pancreatitis has certainly occurred. The change of terminology, unification of definitions and classification was aimed to simplify identification and treatment of acute pancreatitis as well as it's consequences. Intensive conservative treatment is a must in the initial period of the disease.
Interventional treatment should be delayed till the moment of restriction (encapsulation) of pancreatic necrosis.

Interventional treatment regarding the consequences of acute necrotizing pancreatitis should be delayed till the moment of complete limitation of necrosis, its liquidation and rise of WOPN. ${ }^{[9]}$ Mier et al. has proved that conservative treatment 
in the early phase of acute necrotizing pancreatitis and intervention in the later phase can significantly decrease patients' mortality. ${ }^{[11]}$

In 1996, Baron et al. introduced the results of endoscopic treatment of 11 patients with WOPN. ${ }^{[12]}$ After performing the stomy between the lumen of the necrotic collection and the lumen of the gastrointestinal tract in patients, $10 \mathrm{Fr}$ endoprosthesis and $7 \mathrm{Fr}$ nasocystic drains were inserted through the stomy into the cavity of necrosis in order to irrigate the WOPN. ${ }^{[12]}$ Papachristou et al. described transmural drainage of WOPN in 53 patients. ${ }^{[13]}$ A success rate of 45/54 (81\%) and the total number of complications $11 / 53(21 \%)$ were noticed. ${ }^{[13]}$ Smoczynski et al. described the results of effective endoscopic drainage of WOPN in 112 patients. ${ }^{[14]}$ Success in treatment during two years observation was stated in $90.4 \%$ of patients. ${ }^{[14]}$ Complications were found in $25.9 \%$ of patients. ${ }^{[14]}$

Percutaneous drainage of infected pancreatic necrosis was for the first time described in 1998 by Freeny et al., who applied the drainage in 34 patients. ${ }^{[15]}$ Successful treatment was in $47 \%$ of them with a complications' rate of $71 \%$ and mortality of $12 \% .{ }^{[15]}$ On the basis of a meta-analysis of 8 researches considering 286 patients the percutaneous drainage of infected pancreatic necrosis was efficient in $44 \%$ of them. ${ }^{[16]}$ Percutaneous drainage can be exploited together with endoscopic drainage as a method of a multiple approach to the necrotic cavity, ${ }^{[9,14,17]}$ particularly when it comes to penetration of necrosis down the abdominal cavity, up to the minor pelvis. ${ }^{[18]}$ It was proved that the use of percutaneous drainage during endoscopic treatment of WOPN decreases

\section{REFERENCES}

[1] Thoeni RF. The revised Atlanta classification of acute pancreatitis: its importance for the radiologist and its effect on treatment. Radiology. 2012; 262: 751-764. http://dx.doi.org/10.1148/radiol.11 110947

[2] Banks PA, Bollen TL, Dervenis C, et al. Classification of acute pancreatitis-2012: revision of the Atlanta classification and definitions by international consensus. Gut. 2013; 62: 102-111. http: //dx.doi.org/10.1136/gutjnl-2012-302779

[3] Sakorafas GH, Tsiotos GG, Sarr MG. Extrapancreatic necrotizing pancreatitis with viable pancreas: a previously under-appreciated entity. J Am Coll Surg. 1999; 188: 643-648. http://dx.doi .org /10.1016/S1072-7515(99)00045-9

[4] Bradley EL 3rd. A fifteen year experience with open drainage for infected pancreatic necrosis. Surg Gynecol Obstet. 1993; 177: 215-222. PMid:8356492

[5] Nieuwenhuijs VB, Besselink MG, van Minnen LP, et al. Surgical management of acute necrotizing pancreatitis: a 13-year experience and a systematic review. Scand J Gastroenterol Suppl. 2003; 239: the amount of endoscopic and radiological procedures as well as hospitalization time. ${ }^{[17,19]}$ In addition, it increases the efficiency of interventional treatment of WOPN. ${ }^{[17,19]}$

In the described case endoscopic drainage being the only way of access to the necrotic collection was insufficient. On the 27 th day of endoscopic drainage it became necessary to guide the percutaneous drain under radiological imaging. The combination of both minimally invasive methods of treatment of the pancreatic necrosis resulted in complete healing of the patient. As documented, widening the access to the areas of necrosis creates better drainage conditions. The introduced case report describes the process of endoscopic treatment showing it's efficiency in therapy of WOPN, further it's limits coming from access to a collection and making it necessary to exploit additional methods of treatment. In the introduced patient the key to successful treatment of WOPN is the creation of an appropriate irrigation system, enabling aggressive active drainage, and providing passive drainage in the later phase. The endoscopic treatment of WOPN remains an alternative for surgical treatment, ${ }^{[7,13,14]}$ nevertheless surgery still plays an important role in the treatment of pancreatic necrosis, particularly when minimally invasive techniques of treatment became inefficient. ${ }^{[7,20]}$

The therapy for the consequences of acute necrotizing pancreatitis should take place in reference centres, where strict co-operation between a gastroenterologist, endoscopist, radiologist and surgeon is possible. It increases the chances for full recovery of the patients.

\section{CONFLicts OF InTEREST Disclosure}

The authors have declared no conflicts of interest.
111-116. PMid:14743893 http://dx.doi.org/10.1080/00855 920310002799

[6] Howard TJ, Patel JB, Zyromski N, et al. Declining morbidity and mortality rates in the surgical management of pancreatic necrosis. J Gastrointest Surg. 2007; 11: 43-49. PMid:17390185 http: //dx.doi.org/10.1007/s11605-007-0112-4

[7] Freeman ML, Werner J, van Santvoort HC, et al. Interventions for necrotizing pancreatitis: summary of a multidisciplinary consensus conference. Pancreas. 2012; 41: 1176-1194. http://dx.doi .org /10.1097/MPA.0b013e318269c660

[8] Loveday BP, Petrov MS, Connor S, et al. A comprehensive classification of invasive procedures for treating the local complications of acute pancreatitis based on visualization, route, and purpose. Pancreatology. 2011; 11: 406-413. http://dx.doi.org/10.1159/000 328191

[9] Jagielski M. Interventional treatment of pancreatic necrosis. Gastroenetrol Prakt. 2015; 7: 31-42.

[10] van Santvoort HC, Besselink MG, Bakker OJ, et al. Dutch Pancreatitis Study Group. A step-up approach or open necrosectomy for 
necrotizing pancreatitis. New Engl J of Med. 2010; 362: 1491-1502. http://dx.doi.org/10.1056/NEJMoa0908821

[11] Mier J, León EL, Castillo A, et al. Early versus late necrosectomy in severe necrotizing pancreatitis. Am J Surg. 1997; 173: 71-75. http://dx.doi.org/10.1016/S0002-9610(96)00425-4

[12] Baron TH, Thaggard WG, Morgan DE, et al. Endoscopic therapy for organized pancreatic necrosis. Gastroenterology. 1996; 111: 755-764. http://dx.doi.org/10.1053/gast.1996.v111.pm8780582

[13] Papachristou GI, Takahashi N, Chahal P, et al. Peroral endoscopic drainage/debridement of walled-off pancreatic necrosis. Ann Surg. 2007; 245: 943-951. http://dx.doi.org/10.1097/01.sla.00 00254366.19366 .69

[14] Smoczynski M, Marek I, Dubowik M, et al. Endoscopic drainage/debridement of walled-off pancreatic necrosis-single center experience of 112 cases. Pancreatology. 2014; 14: 137-142. http://dx.doi.org/10.1016/j.pan.2013.11.005

[15] Freeny PC, Hauptmann E, Althaus SJ, et al. Percutaneous CT-guided catheter drainage of infected acute necrotizing pancreatitis: techniques and results. AJR Am J Roentgenol. 1998; 170: 969-975. PMid:9530046 http://dx.doi.org/10.2214/ajr.170.4.953 0046
[16] Bello B, Matthews JB. Minimally invasive treatment of pancreatic necrosis. World J Gastroenterol. 2012; 18: 6829-6835. http: //dx.doi.org/10.3748/wjg.v18.i46.6829

[17] Ross A, Gluck M, Irani S, et al. Combined endoscopic and percutaneous drainage of organized pancreatic necrosis. Gastrointest Endosc. 2010; 71: 79-84. http://dx.doi.org/10.1016/j.gie .2009 .06 .037

[18] Raczynski S, Teich N, Borte G, et al. Percutaneous transgastric irrigation drainage in combination with endoscopic necrosectomy in necrotizing pancreatitis (with videos). Gastrointest Endosc. 2006; 64: 420-424. PMid:16923493 http://dx.doi.org/10.1016/j.gie .2006 .02 .052

[19] Gluck M, Ross A, Irani S, et al. Dual modality drainage for symptomatic walled-off pancreatic necrosis reduces length of hospitalization, radiological procedures, and number of endoscopies compared to standard percutaneous drainage. J Gastrointest Surg. 2012; 16 : 248-256. http://dx.doi.org/10.1007/s11605-011-1759-4

[20] Stamatakos M, Stefanaki C, Kontzoglou K, et al. Walled off pancreatic necrosis. World J Gastroenterol. 2010; 16: 1701-1712. http://dx.doi.org/10.3748/wjg.v16.i14.1707 